\title{
Investigation of free convection in the cubic cave with PIV and POD methods
}

\author{
D.A. Sergeev ${ }^{1}$, A.A. Kandaurov² \\ A Institute of Applied Physics RAS \\ B Nizhny Novgorod State Technical University n.a. R.E. Alekseev \\ ${ }^{1}$ ORCID: oooo-ooo3-4910-3935, daniil@appl.sci-nnov.ru \\ 2 ORCID: 0000-0002-0014-8887,green.pb@gmail.com
}

\begin{abstract}
Investigations of the flow structure on the benchmark "cube" under conditions of free convection caused by a vertical temperature gradient are performed using a low cost PIVmeasuring system with continuous laser. The obtained velocity fields were processed with POD method to obtain typical structures (modes) in the flow. It is shown that the steady flow can be described by combination of the first three modes. It is demonstrated that only starting with the third mode there is a significant change in the spectra of the amplitude coefficients of the modes, the peak shifts to the region of higher frequencies. Thus accounting for this mode is essential for describing turbulent processes in the system and verification of numerical calculations.
\end{abstract}

Keywords: verification CFD, free convection, velocity fields, PIV, POD.

\section{Abstract}

Numerical simulation of temperature and velocity fields of turbulent non-isothermal flows is an important and useful in the studies of processes in power plants, including nuclear (NPP). Temperature fluctuations arising in turbulent non-isothermal flows (in comparison with laminar), lead to additional cyclic thermal strains on the walls of the equipment and in some cases significantly reduce the service period of both individual equipment and the facilities in a whole. In addition, non-stationary non-isothermal flows have a significant impact on the output of temperature gauges, which are used to monitor and control processes on the NPP.

We consider as the reference the International Working Group of Experts CFD4NRS (uniting experts in the field of NPP safety studies) experiment to verify numerical modeling recommended the studying natural convection inside a cubic cavity with a given vertical temperature gradient. Here, it should also be noted that natural convection in closed cavities is a traditional object of basic research, due to the possibility of implementing various flow regimes under similar conditions [1,2].

The studying of natural convection in closed cavities of various configurations is one of the main procedures in modeling various convective processes in engineering applications. The main part of the research in this area is devoted to the study of the convection in the rectangular cavities with the temperature differences in the horizontal plane (heating and cooling on the side walls).

The studies with a vertical temperature gradient are more interesting when the complex process-time structure of large-scale flows are considered. Those flows can be obtained under these conditions. Most of these studies were performed for cylindrical [3] and cubic cavities $[5,6]$. Numerical calculations of convection in a cubic cavity with a vertical temperature difference were performed for small and moderate values of the Rayleigh number $\left(3 \cdot 5 \cdot 10^{3} \leq \mathrm{Ra}\right.$ 
$\left.\leq 6 \cdot 10^{4}\right)$ [3-6]. It was demonstrated that even for small values of supercriticality in laminar regimes, the formation of turbulent flows of various spatial structures is possible.

Of great interest for the above-mentioned practical applications associated with modeling processes at NPP are the convective flows with developed turbulence at $10^{8} \leq \mathrm{Ra} \leq 101^{3}$ and higher. However, such regimes have been studied quite a little. The reason for this is that the evolution of large-scale flows in turbulent regimes proceeds rather slowly, and, as experiments show, the description of the evolution of large-scale structures takes from several to tens of hours. Performing numerical simulations on such long time intervals is a non-trivial task and requires careful selection of a numerical model and the use of a high-performance computing system. Thus, the procedure for verifying numerical calculations becomes important.

It is necessary to provide the most representative comparison of its results with the available experimental data within verifying the results of numerical simulation. The comparison of instantaneous or in contrast average flow characteristics, including velocity fields, does not allow one to properly verify the results of numerical calculations in terms of time evolution. On the other hand, a comparison of the time spectra obtained at control points may also not to be comprehensive. The representativeness of the choice of their position can be obtained only taking into account the spatial structure of the flow. In this case, the use of the Proper Orthogonal Decomposition (POD) method can help, which, on the one hand, makes it possible to show the main spatial characteristics of the flow, and on the other, to obtain their temporal spectral characteristics.

\section{Scheme of experiment PIV-method use.}

The benchmark was a cube with a side of $25 \mathrm{~cm}$ made of optical glass with a thickness of 5 $\mathrm{mm}$, completely filled with fresh water. , We provided a special stabilized system of electrical heating of the bottom with feedback on temperature gauges in order to maintain a constant temperature difference of up to 20 degrees between the upper and lower bound. The corresponding Rayleigh number in the experiment is $\mathrm{Ra}=4 \cdot 4 \cdot 10^{9}$ (which corresponds approximately to the middle of the range of values of interest in modeling real conditions, see above). Fig. 1 shows the general scheme of the experiment and a separate illustrative frame obtained with a long exposure (3 seconds).
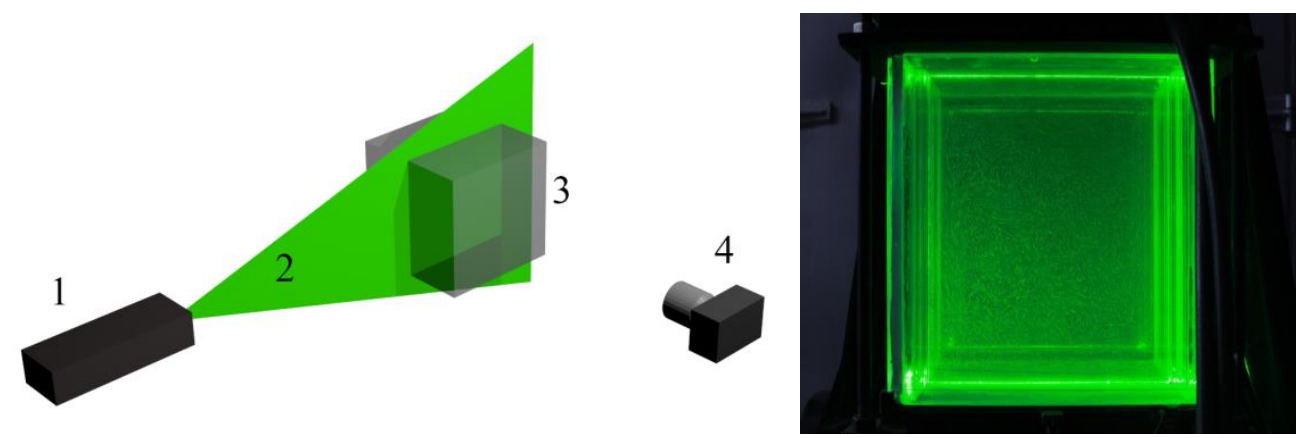

Fig. 1. Left: principal scheme of experiment: 1- laser, 2 - laser sheet, 3 - cub with water, 4 camera. Right: The obtained with long exposition image, that demonstrates large scale structures in the flow

To measure the fields of the flow rate of natural convection due to the vertical temperature gradient, the PIV method was used. The studies were carried out according to the simplest scheme with a continuous laser illumination of a LCS-DTL-413 diode-pumped green laser (1.5 W, $527 \mathrm{~nm})$. A vertical laser sheet was formed from a beam using a defocusing cylindrical lens (2.5 mm radius). Microparticles (HGS hollow glass beads) with a diameter of 10 $\mu \mathrm{m}$ were used to visualize the flow. The filming was performed with a Canon EOS 5D Mark II serial camera with a CMOS matrix of $21.1 \mathrm{Mpx}$ (the scale of the resulting image is $240 \mu \mathrm{m} /$ px). Frame resolution 19201080 with a frequency of 30 frames / second, and shutter time of 
$30 \mathrm{~ms}$. Comparison with the results obtained for the Dantec serial 2D-PIV system under the same conditions demonstrated that this simplified system allow to measure velocity fluctuations with a time resolution from 0.001 to $15 \mathrm{~Hz}$ and accuracy not worse than $0.1 \mathrm{~mm} / \mathrm{s}$ in the range of velocities up to $2 \mathrm{~cm} / \mathrm{s}$ at the selected filming scaling.

\section{Data processing, retrieving velocity fields and analysis.}

3200 seconds of video were processed by PIV-method totally. Cross-correlation processing for pairs of sequent frames was performed. The interrogation window was $32^{\prime} 32$ pixels, overlapping 50\%. Thus interval on coordinate grid of velocity field was 16 pixels or $3.8 \mathrm{~mm}$. An adaptive scheme for searching for a cross-correlation function with a Gaussian approximation by three points of its peak was used to ensure sub-pixel accuracy, similar to that we used earlier in [6]. The resulting velocity fields were filtered by a 10-fold downsampling sample in time. This was done using averaging with a 10-frame sliding window. For the window containing less than 30\% of unfiltered velocity values the linear time interpolation was performed instead of averaged value calculation. As a result, all measured velocity fields were filtered and combined into a three-dimensional array containing 61'62'9587 points, in each two velocity components were measured.

Based on the results of processing this array, we obtained average velocity fields and fields of deviation for each of the velocity components (see Fig. 2). As it was expected, it has demonstrated the strongest variations in the near-wall regions. Comparison of the velocity fields obtained by averaging over various time intervals (see Fig. 3) demonstrates the absence of any significant changes at times exceeding 60 seconds, which gives a lower estimate for the time during which the numerical count, the results of which can be compared with the data of the present experiment, if we consider it as a reference.

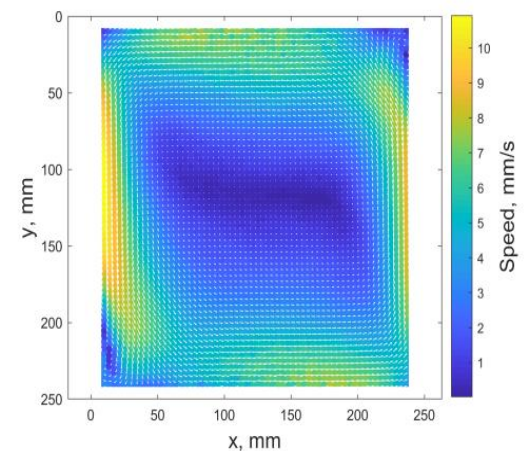

a)

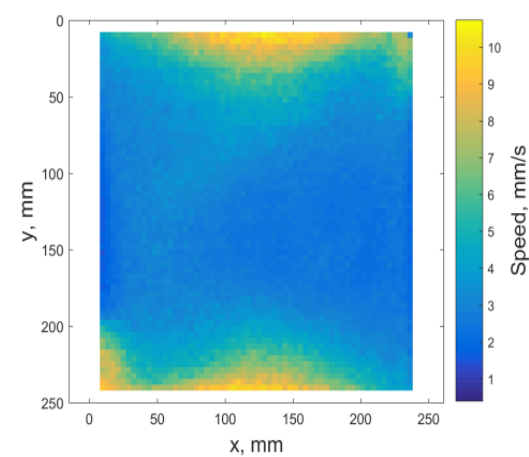

б)

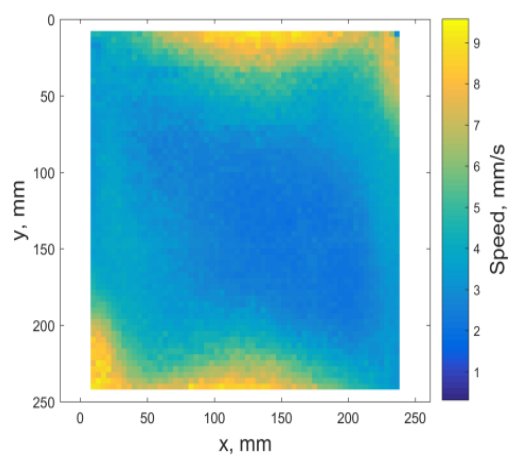

B)

Fig. 2. Average velocity field (top) and a field of standard deviations for horizontal (middle) and vertical (bottom) velocity components. Color scale indicate m/s. 

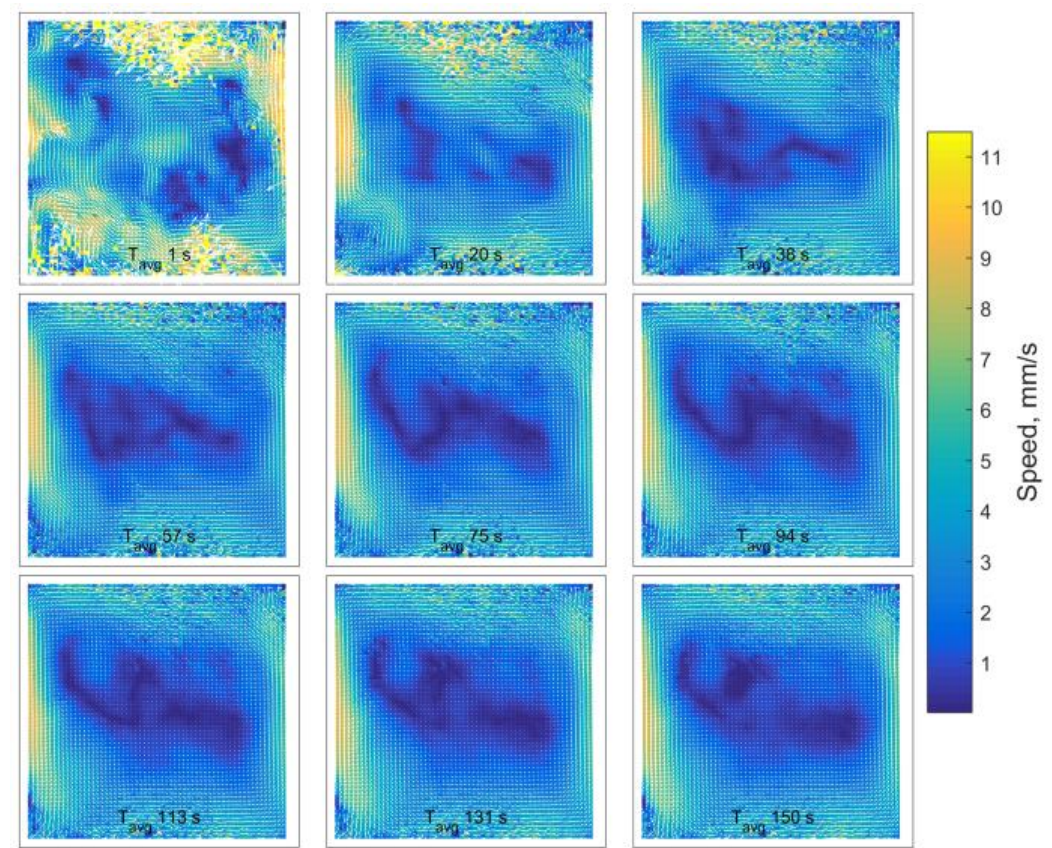

Fig. 3. Velocity fields for a set of 9 different averaging times from 1 to 150 seconds (bottom).

\section{Procedure and results of POD-method using}

The high spatial resolution of the obtained velocity fields made it possible to successfully use the Proper orthogonal decomposition (POD) method to isolate the basic restore modes of the flow.

The results of using POD in processing velocity fields obtained by PIV-methods can be found in the relatively recently published papers (see [8-11]). For example, in [8], POD was used to obtain the phase-averaged statistical characteristics of the turbulent wake behind the bodies. Interpretation of the data obtained in the short series of PIV-measurements in a diesel model with a high temporal resolution using POD method is described in [9]. In [10] POD was used to study the relationship between the characteristics of power spectra with the observed structures in a flow over an open cavity. In [11], POD was used to reconstruct threedimensional flow structures and study their temporal evolution in a turbulent jet in a transverse flow. To analyze the velocity fields, we used the so-called "shapshot POD" technique, in the variant described in [11]. The main idea of the POD algorithm is to represent the instantaneous velocity fields as a sum of modes with time-dependent coefficients. To find those modes from ansamble of $\mathrm{N}$ velocity fields, the following representation in form of matrix $l \times m$ of fluctuating parts of both velocity componens is used:

$$
\mathrm{U}=\left[\begin{array}{lll}
\mathbf{u}^{1} \mathbf{u}^{2} \ldots \mathbf{u}^{N}
\end{array}\right]=\left[\begin{array}{cccc}
u_{1}^{1} & u_{1}^{2} & \cdots & u_{1}^{N} \\
\vdots & \vdots & \vdots & \vdots \\
u_{s n}^{1} & u_{m m}^{2} & \cdots & u_{j m}^{N} \\
v_{1}^{1} & v_{1}^{2} & \cdots & v_{1}^{N} \\
\vdots & \vdots & \vdots & \vdots \\
v_{s m}^{1} & v_{b m}^{2} & \cdots & v_{s m}^{N}
\end{array}\right],
$$

$u$ and $v$ here are fluctuating parts of the horizontal and vertical components correspondingly, and the superscript of the velocity indicates the number of the velocity field in the sequence. Than the matrix $\tilde{\mathrm{C}}=\mathrm{U}^{T} \mathrm{U}$ is calculated and corresponding eigenvalue problem $\tilde{\mathrm{C}} \mathrm{A}^{i}=\lambda^{i} \mathrm{~A}^{i}$ is solved. The solutions are arranged by eigenvalue as: $\lambda_{1}>\lambda_{2}>\ldots>\lambda_{N}=0$. And POD modes are found as: 


$$
\phi^{i}=\frac{\sum_{n=1}^{N} A_{n}^{i} \mathbf{u}^{n}}{\left\|\sum_{n=1}^{N} A_{n}^{i} \mathbf{u}^{n}\right\|}, i=1, \ldots, N
$$

With POD modes arranged as $\Psi=\left[\phi^{1} \phi^{2} \ldots \phi^{N}\right]$ decomposition coefficients can be found for the $e^{n}$ velocity field as $\mathbf{a}^{n}=\Psi^{T} \mathbf{u}^{n}$. A fluctuating part of velocity field can be reconstructed as:

$$
\mathbf{u}^{n}=\sum_{i=1}^{N} a_{i}^{n} \phi^{i}=\Psi \mathbf{a}^{n} .
$$

The obtained velocity fields were processed with POD method to obtain typical structures (modes) in the flow (Fig. 4). It was shown that the steady flow can be described by combination of the mean flow and the first three modes (up to $95 \%$ of the kinetic energy of the flow is contained in them). It was demonstrated that only starting with the third mode there is a significant change in the spectra of the amplitude coefficients of the modes, the peak shifts to the region of higher frequencies to $0.02 \mathrm{~Hz}$ in comparison with the first two modes (0.006 Hz) (Fig. 5).
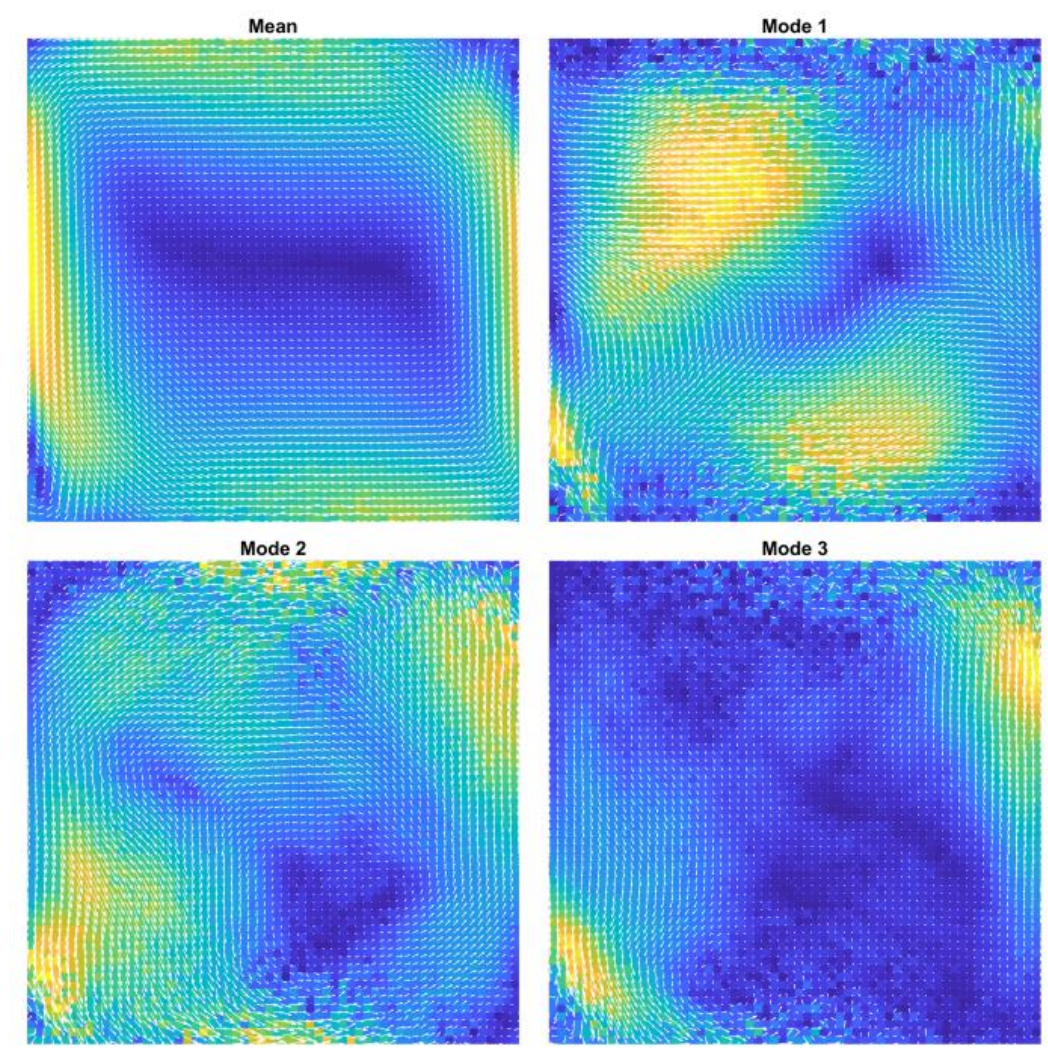

Fig. 4. Mean velocity field and 4 main POD modes, arranged by descending energy. 

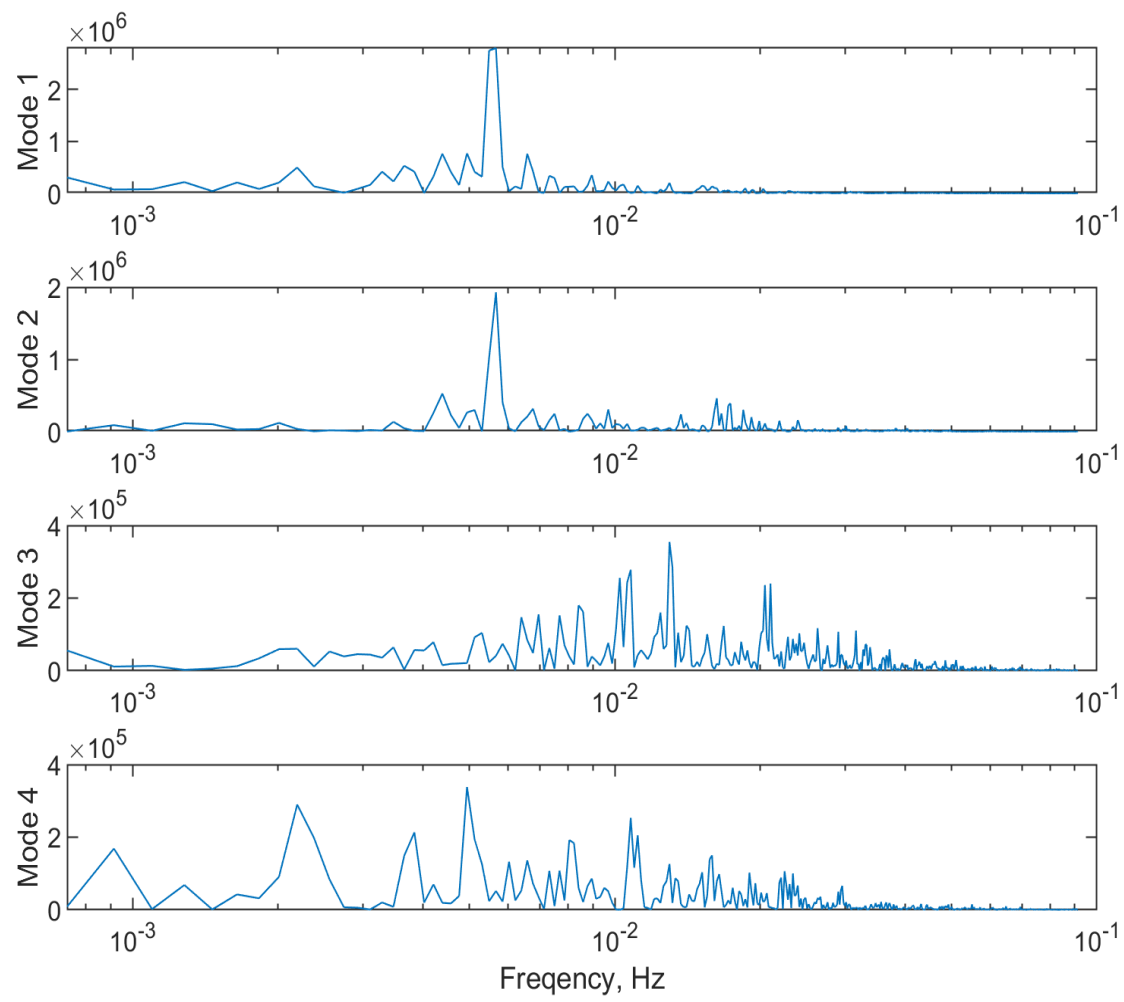

Fig.5. Spectra obtained from time dependences coefficients for the four modes (are listed from top to bottom in descending order of average kinetic energy of fluctuations).

\section{Conclusion}

Experimental studies of natural convection processes on the benchmark "cube" were performed for the verification problems of CFD processes in NPP. The experiments were performed under a constant vertical temperature gradient with a Rayleigh number of $4.4 \cdot 10^{9}$, which corresponds to turbulent flow regimes. PIV system based on the simplest scheme with continuous illumination was used for measurements. For the measured velocity field sequences, the POD method procedure was used to develop the most representative verification methods for numerical CFD calculations. It is shown that more than 95\% of the kinetic energy of turbulent fluctuations is determined by the combination of the first three modes. Thus, a comparison with the results of CFD is reasonable to carry out on the average, as well as on the spectral characteristics of these modes.

This work was partial supported by the Russian Foundation of Basic Research No. 18-48520023 (providing numerical simulations) and Russian Science Foundation No. 18-19-00473 (providing measurements).

\section{References}

1. G. Gershun, E. Zhuhovitsky, A. Nepomnyashiy, Stability of convective currents. M.: Science. 1989.

2. V. Zimin, P. Frik, Turulent convection, M.: Science. 1988.

3. Ozoe H., Yamamoto K., Churchill S.W., Sayama H. Three-dimensional, numerical analysis of laminar natural convection in a confined fluid heated from below // J. Heat Trans. T. ASME, 1976, V. 98, N. 2, P.202-207.

4. Hernández R., Frederick R.L. Spatial and thermal features of three dimensional RayleighBénard convection // Int. J. Heat Mass Tran., 1994, V. 37, N. 3, P. 411-424. 
5. Pallarés J., Cuesta I., Grau F.X., Giralt F. Natural convection in a cubical cavity heated from below at low Rayleigh numbers // Int. J. Heat Mass Tran., 1996, V. 39, N. 15, P. 32333247.

6. Pallarés J., Grau F.X., Giralt F. Flow transitions in laminar Rayleigh-Bénard convection in a cubical cavity at moderate Rayleigh numbers // Int. J. Heat Mass Tran., 1999, V. 42, N. 4, P. 753-769.

7. Sergeev D.A. A Measuring System for Studying Liquid Flowsby the Particle Image Velocimetry Method Based on a Diode-Pumped Solid-State Laser // Instruments and Experimental Techniques, 2009, V. 52, N. 3, P. 1-7.

8. Perrin R., Braza M., Cid E., Cazin S., Barthet A., Sevrain A., Mockett C., Thiele F. Obtaining phase averaged turbulence properties in the near wake of a circular cylinder at high Reynolds number using POD // Exp. in Fluids, 2007, V. 43, I. 2, P.341-355.

9. Cosadia I., Borée J., Dumont P. Coupling time-resolved PIV flow-fields and phaseinvariant proper orthogonal decomposition for the description of the parameters space in a Diesel transparent engine // Exp. in Fluids, 2007, V. 43, I. 2, P.357-370.

10. Pastur L., Lusseyran F., Fraigneau Y., Podvin B. Determining the spectral signature of spatial coherent structures in an open cavity flow // Physical Review E, 2005, V. 72, 065301.

11. Meyer K.E., Pedersen J.M., Özcan O. A turbulent jet in crossflow analyzed with proper orthogonal decomposition. // J. Fluid Mech., 2007, V. 583, P. 199-227. 